\title{
Special issue on Intelligent software services for loT and edge computing
}

\author{
Jian $\mathrm{Yu}^{1}$. Sira Yongchareon ${ }^{1}$ - Seyed Ali Ghorashi ${ }^{2}$ - Dongjin $\mathrm{Yu}^{3}$
}

Published online: 23 February 2022

(c) The Author(s), under exclusive licence to Springer Nature Switzerland AG 2022

\section{Introduction}

Intelligent software services for IoT and edge computing is an important research area for the future ubiquitous software systems and services including IoT and edge computing. Given the recent advances in heterogeneous sensor networks and cloud services, this research area is evolving from the traditional distributed computer systems and Internet of Things into intelligent edge computing. This future network computing will provide an environment where everyday physical objects such as buildings, sidewalks, and commodities are readable, recognizable, addressable, and even controllable using services via intelligent software provided through the Internet. The capability of integrating the information from both the physical world and the virtual one not only affects the way how we live, but also creates tremendous new business opportunities such as smart healthcare, intelligent traffic management, efficient supply chains, and improved environmental monitoring.

This special issue has included five articles representing some of the state-of-the-art developments in IoT and edge computing-related intelligent software services. The first paper by Yang et al. entitled "MaritimeDS: A Data Service Framework for Unsupervised Maritime Traffic Monitoring based on Trajectory Big Data" proposes a novel layered model for maritime traffic data services. Based on the service model, an unsupervised learning method is designed

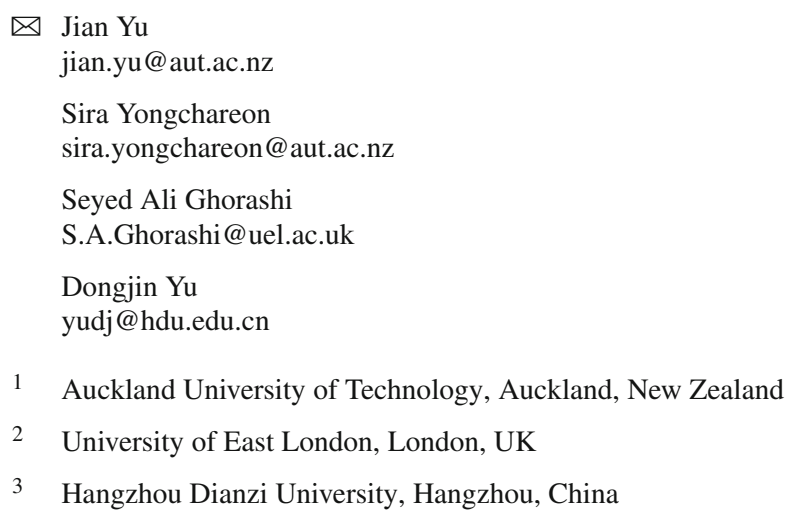

and implemented for maritime networks extraction. The second paper by Almasri et al. entitled "Multi-Objective Optimization of Task Assignment in Distributed Mobile Edge Computing" proposes a multi-objective optimization solution for tasks assignment of edge devices where both the energy consumption of edge devices and the computation time of tasks are minimized. A novel multi-objective evolutionary algorithm is proposed for the optimization. The third paper by Zhang et al. entitled "Task-load Aware and Predictive-based Workflow Scheduling in Cloud-edge Collaborative Environment" proposes a predictive-based method to deal with dynamic workflow scheduling of IoT data processing tasks in edge computing that utilizes historical solutions to generate candidate solutions to accelerate population optimization and reduce the total time of scheduling. We have also included two survey papers: one by Chen et al. on the topic of "Machine Learning Methods for Hospital Readmission Prediction", and one by Amraoui and Zouari on the topic of "Securing the Operation of Smart Home Systems".

The guest editors would like to take this opportunity to thank all the authors for the efforts they put in the preparation of their manuscripts and for their valuable contributions. We wish to express our deepest gratitude to the referees who provided very useful and thoughtful feedback to the authors. Finally, our sincere thanks go to the Editor-in-Chief, Professor Juan Carlos Augusto and Antonio Coronato, of Journal of Reliable Intelligent Environments for his kind support, advice, and encouragements throughout the preparation of this special issue.

Publisher's Note Springer Nature remains neutral with regard to jurisdictional claims in published maps and institutional affiliations. 